\title{
Guiding the reorganization of motor redundancy for assistance and rehabilitation after spinal cord injury
}

\author{
Dalia De Santis, Ferdinando A. Mussa-Ivaldi
}

\begin{abstract}
We discuss the strategies that the motor system may adopt while learning to control a body-machine interface (BMI) and how a novel approach to modulate the parameters of the BMI could be effective in facilitating learning of an appropriate feedforward control strategy. The same mechanism could potentially be used for teaching different control strategies to the user.
\end{abstract}

\section{INTRODUCTION}

High-level injuries to the spinal cord compromise function of the upper limb and result in severe deterioration of the quality of life. Rehabilitative training, such as exercise therapy, functional stimulation or training mediated by Human-Machine interfaces can improve upper limb strength and function and contributes to restoring independence. However, the functional recovery is strictly dependent on the amount of damage to the neural circuitry which, in time, can lead to progressive and permanent structural as well as functional changes in the CNS. Recent evidence suggests that some of these modifications might not be irreversible and that CNS remodeling is dependent upon the engagement of muscle groups in skilled activities [1]. Moreover, the acquisition of motor skills seems to have a very distinctive signature on corticospinal plasticity that is not observed during passive repetition of movements or simple strength training [2], [3].

The fact that plasticity is generally confined to the group of muscles that was engaged in learning the sensorimotor skills, raises the question of how to involve in skilled activities movements or muscles for which residual voluntary control is severely limited by weakness or paralysis. A particular category of non-invasive HMIs that make use of wearable sensors, called Body-Machine Interfaces (BMI's), was designed with the purpose of identifying movements that were still available to the paralyzed user and remapping them into a control input to a wide range of assistive devices [4], [5]. The general function

Research supported by NIDRR grant H133E120010 and NICHD grant 1R01HD072080. Results incorporated in this manuscript have received funding from the European Union's Horizon 2020 research and innovation program under the Marie Sklodowska-Curie, project REBoT, G.A. No750464.

D. De Santis is with Northwestern University, the Shirley Ryan AbilityLab, Chicago IL 60611 USA, and the Istituto Italiano di tecnologia, Genova 16152 IT (corresponding author: dalia.desantis@northwestern.edu)

F. A. Mussa-Ivaldi is with Northwestern and the Shirley Ryan AbilityLab (sandro@northwestern.edu) implemented by a BMI is of reducing the dimensionality of the input signals coming from movement of the user in order to define a manifold used to command systems external to the body that are generally of much lower dimension.

The advantage of the BMI systems is that the mapping can be tailored to the (dis)abilities of the user. The disadvantage, is that the burden of learning a suitable set of body postures or coordinated movements that actually can be used in controlling the system is left to the user. While this learning challenge is likely be one of the key ingredients to the formation of a new motor repertoire, it could also constitute a strong factor of frustration and withdrawal from the use of the interface.

Here, we briefly discuss the strategies that the motor system may adopt while learning to control a BMI and how a novel approach to modulate the parameters of the BMI could be effective in facilitating learning of an appropriate feedforward control strategy. Much of this discussion applies also to brain-machine interfaces.

\section{MATERIALS AND METHODS}

\section{A. Learning when a problem has infinite solutions}

Let us define A the (linear) mapping from a high to low dimensional space, where $\boldsymbol{p} \in V^{m}$ is the vector of control signals and $\boldsymbol{q} \in V^{n}$ is the n-dimensional vector representing the configuration of the body signals, with $\mathrm{m}<\mathrm{n}$, such that $\boldsymbol{p}=\boldsymbol{A} \boldsymbol{q}$. In particular, the mapping is a linear transformation obtained by projecting the input signal $\boldsymbol{q}$ along the directions in which the user expresses the greatest mobility.

The learner is confronted with two problems when attempting to control the interface for the first time. The first, relates to finding an appropriate subspace of body configurations (or muscle activations) that lies at least partially on the linear manifold defined by A. Following Braun et al. [6], we can call this the structural problem. The second, relates to how to perform a specific task in a proficient way. We could call this latter the performance problem. Finding a solution to the structural problem, that is finding movement patterns that share some dimension with the BMI manifold, is a necessary condition for the user to solve the performance problem efficiently. However, having to concurrently perform a goal-directed task introduces constraints in the way the structural problem is solved. This implies that the solution identified by the user might differ depending on the individual task [7]. 


\section{B. Strategy for adapting the map}

We hypothesized that, once BMI users have identified a suitable movement manifold for controlling the interface, learning in terms of improving movement performance would proceed fast. Therefore, we devised a strategy for facilitating the identification of the BMI manifold through its online reorientation to better match the movement manifold of the user. For doing so, we implemented an online PCA algorithm [8] that performs a series of stochastic estimates of the eigenvectors of the movement covariance matrix while the subject is attempting to perform a task. The estimate at the current time stamp $\mathrm{k}$ is composed of a memory element (the value of the eigenvector $\mathbf{v}_{\mathbf{1}}$ at the previous step) and a novel element that depends on the mismatch between the sample covariance and the axes of the map and defines the direction of attraction of $\mathbf{v}_{\mathbf{1}}$ :

$$
\mathbf{v}_{\mathbf{1}}{ }^{(k)}=(1-\eta) \mathbf{v}_{\mathbf{1}}{ }^{(k-1)}+\eta\left(\boldsymbol{s}^{(k)} \boldsymbol{s}^{(k) T} \cdot \frac{\mathbf{v}_{\mathbf{1}}{ }^{(k-1)}}{\left\|\mathbf{v}_{\mathbf{1}}{ }^{(k-1)}\right\|}\right)
$$

$\eta$ is the rate of update and weights the influence of new samples on the map. The effect of this computation is a progressive rotation of the original map towards the estimated directions of variance expressed by the user. For good values of $\eta$, the algorithm should be bound to converge to the subject's manifold. At the equilibrium, a reversed approach could be used to promote the adoption of specific control strategies by the user.

\section{Experimental Methods}

Healthy participants practiced controlling a BMI that collected movement data of the arms via 4 IMU sensors (placed bilaterally, one on the arm above the biceps and one on the ulnar bone below to the elbow) for a total of 8 angular measurements (Pitch and Roll of each sensor). The BMI map was computed via PCA over the sensor data collected during 1 minute of random movements of the arms (task 1). Participants were first asked to train in a reaching task with a cursor on a screen for 20 minutes (task 2) and then to track the movement of a target on the screen (task 3 ).

During the reaching practice, we quantified performance over trials in terms of the time they needed to reach a target. Since we were interested in understanding how the participants shaped their movement distribution following practice with the map, we quantified the relative similarity of two (normal) distribution of sensor values by the Kullback-Leibler divergence. Finally, we computed the planarity index (PI) as the variance accounted for by the first 2 principal components of the sensor values over a certain period of time. Given that the dimensionality of the task is only 2, we expected our participants to increase planarity in time if they expressed structural learning.

\section{RESULTS}

Figure 1 shows that the time course of the reaching performance (reaching time, z-transformed) and the development of a movement manifold (planarity index and z-transformed KL divergence) in the reaching task could be well summarized by a process with two time-scales. The fast component accounts for the changes in the very first

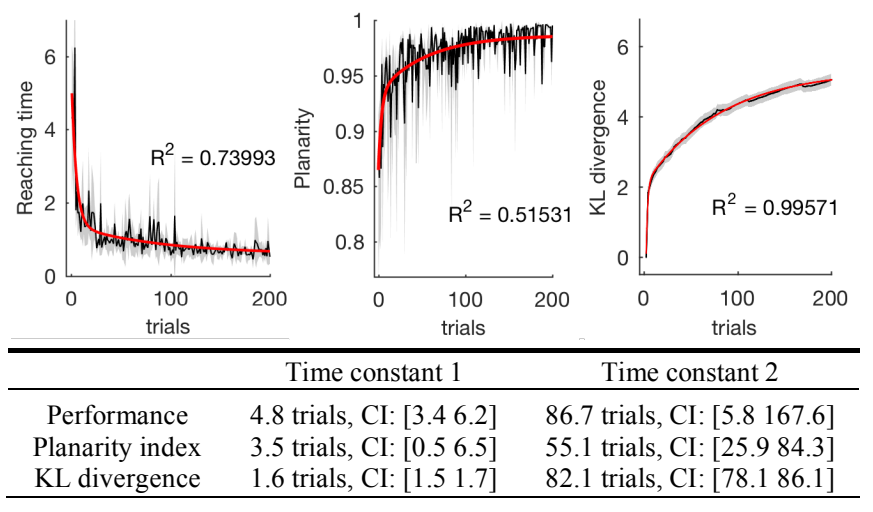

Fig 1. Average values of reaching time and KL divergence over 8 subjects across reaching trials (black) and fitting via a double exponential function (red): values have been $\mathrm{z}$-transformed. KL divergence is relative to the movement distribution on trial 1 .
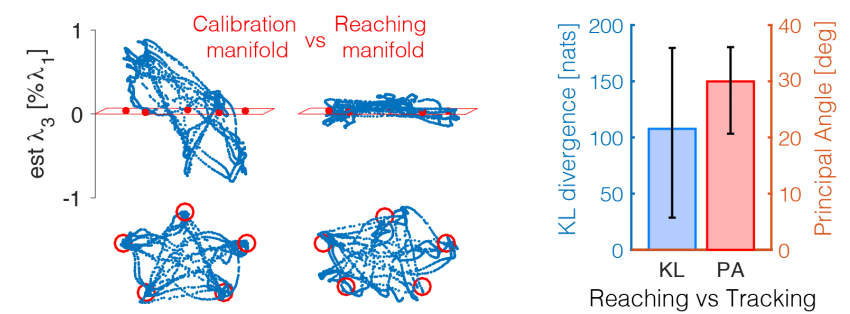

Fig 2. Movement manifold dependency on the task. Left-(3D top, 2D bottom) projection of the sensor values onto the calibration manifold (left) vs. the manifold obtained by PCA decomposition during reaching (right). Right - difference between movement distributions (KL divergence) and 2D manifolds (principal angle) during reaching vs. tracking.

trials, while a late component tends to appear after about 50 trials. In particular, Figure 1 seems to suggest that significant improvements in performance are second to the identification of a suitable movement manifold, which tends to have the same dimension of the task. As anticipated, the movement manifold was task dependent. Figure 2, left panel, exemplifies the difference between the movement manifold during calibration and during reaching, while the right panel shows the difference during reaching and tracking.

\section{REFERENCES}

[1] X. Lu, C. R. Battistuzzo, M. Zoghi, and M. P. Galea, "Effects of training on upper limb function after cervical spinal cord injury: a systematic review.," Clin. Rehabil., vol. 29, no. 1, pp. 3-13, Jan. 2015.

[2] M. Lotze, C. Braun, N. Birbaumer, S. Anders, and L. G. Cohen, "Motor learning elicited by voluntary drive," Brain, vol. 126, no. 4, pp. 866-72, Apr. 2003.

[3] J. L. Jensen, P. C. D. Marstrand, and J. B. Nielsen, "Motor skill training and strength training are associated with different plastic changes in the central nervous system.," J. Appl. Physiol., vol. 99, no. 4, pp. 1558-68, Oct. 2005.

[4] E. B. Thorp et al., "Upper Body-Based Power Wheelchair Control Interface for Individuals with Tetraplegia.," IEEE Trans. Neural Syst. Rehabil. Eng., vol. PP, no. 99, p. 1, Jun. 2015.

[5] F. Abdollahi et al., "Body-Machine Interface Enables People With Cervical Spinal Cord Injury to Control Devices With Available Body Movements: Proof of Concept," Neurorehabil. Neural Repair, vol. 31, no. 5, pp. 487-493, May 2017.

[6] D. A. Braun, C. Mehring, and D. M. Wolpert, "Structure learning in action," Behav. Brain Res., vol. 206, no. 2, pp. 157-165, 2010.

[7] C. Pierella et al., "Learning new movements after paralysis: Results from a home-based study," Sci. Rep., vol. 7, no. 1, p. 4779, 2017.

[8] J. Weng, Y. Zhang, and W. S. Hwang, "Candid covariance-free incremental principal component analysis," IEEE Trans. Pattern Anal. Mach. Intell., vol. 25, no. 8, pp. 1034-1040, 2003. 